Journal of Computer Science 7 (1): 90-94, 2011

ISSN 1549-3636

(C) 2011 Science Publications

\title{
A Fast Handover Scheme for Multicasting in IPv6 based Mobile Ad hoc Networks
}

\author{
${ }^{1}$ H. Parveen Sultana, ${ }^{2}$ M. Pounambal and ${ }^{1}$ Dr. P. Venkata Krishna \\ ${ }^{1}$ Department of Computing Science and Engineering, VIT University, \\ Vellore, Tamil Nadu, India \\ ${ }^{2}$ Department of Information Technology and Engineering, VIT University, \\ Vellore, Tamil Nadu, India
}

\begin{abstract}
Problem statement: In the previous researches, steps were taken to resolve the problems of multicasting, after several discussions. Actually these issues were raised, while multicasting packets from Internet Protocol (IP) to Mobile Nodes (MN). On the other hand, there is very little concern about the problem of packet loss reduction. Sometimes the occurrence of multicast service chaos is ignored during handovers. Therefore in this study it has been tried to explain the optimal multicast technique for Mobile IPv6 (MIPv6) to diminish the required amendment to the existing fast handover. Approach: An alternate method is suggested in Fast handover for MIPv6 (FMIPv6) to lessen the packet losses during handovers, before tunneling. Based on the qualities of the multicast subscription techniques for the Mobile IPv6 (MIPv6), multicast upholds method for FMIPv6. MIPv6 in general comprise two types of multicasting techniques, which are related to Home Agent (HA) and Foreign Agent (FA). These techniques help the MN to obtain the packets in roaming location referred as Foreign Network (FN), which is being forwarded by the Core Network (CN). But due to the handover latency problem MN have to experience packet loss while switching between any two Access Routers (AR). A protocol has been designed to conquer the issue of packet loss in MIPv6. Results and Conclusion: This protocol can be used during joining process in MIPv6 before tunneling to eliminate the IP connectivity time. This implementation allows a mobile node to be connected more quickly at a fresh point of connection, when that mobile node moves with less packet losses.
\end{abstract}

Key words: Multicast, mobile node, FMIPv6, Mobile Nodes (MN), Home Agent (HA), Foreign Network (FN), Third Generation Partnership Project (3GPP), Access Routers (AR), Internet Engineering Task Force (IETF), Core Network (CN)

\section{INTRODUCTION}

From the previous century to the present, a huge range of wireless technologies has resulted in a rapid growth of wireless connections like Satellite and WiMAX and Mobile technologies like Bluetooth, WiFi (Kwon et al., 2008). The combination of these wireless communications (Kwon et al., 2008) provides worldwide access to wireless. A few countries like the United Kingdom, India and the United States have the best wireless corporations, which provide various networking technologies. These connectivity technologies have been adopted in various fields to offer uninterrupted networking services. The Third Generation Partnership Project (3GPP) (Nguyen, 2008) and Unlicensed Mobile Access (UMA) (Nguyen, 2008) have recommended these wireless technologies, which give seamless services during handovers.
In networking, Multicast (Paul, 1998; Gattupalli and Krishna, 2010) is a way to deliver information to a group of destination nodes in a single transmission simultaneously from the source node. It is also one of the types of wireless communication. In multicasting, the data is conveyed on a network link and messages are delivered over each network connection only once. The messages are reproduced at the destination points. Due to dynamic changes in the connections and due to the significance of mobility in the Internet Mobile IP (Suh et al., 2001; Louvros, 2007), a routing protocol (Pack and Choi, 2003; Gattupalli and Krishna, 2010) is created. This routing protocol provides seamless communication between the source and the destination nodes. Mobile IP use many ways like Mobile with packet Internet, Video Conferencing and LED television with Internet to relay information across an internetwork. In this multimedia group messages can be conveyed by either one-to-many (Kwon et al., 2008) or 
many-to-many communications (Kwon et al., 2008). Mobile IP (Suh et al., 2001; Alfawaer et al., 2007) provides users with uninterrupted facility to get connected across the network. Mobile nodes are linked with the network by means of it IP addresses. They switch to another network by changing IP address. The fundamental process of exchanging information between two networks is achieved through mobile IP by users, which is also translucent for a user. Many routing protocols related to mesh and ad hoc networks have been developed by the network research society (Kwon et al., 2008), to create well-ordered multicasting to unidentified destinations. But these routing protocols perform inefficiently in networking environments. Because these routing protocols are created assuming that, sometime in the networking range multicast senders and receivers are passive.

Mobile IP has only been an add-on (Suh et al., 2001; Alfawaer et al., 2007), but the new communication protocol IPv6 has overcome restriction and solved many problems raised by mobile IP. To resolve these problems, the current Internet Engineering Task Force (IETF) (Kwon et al., 2008), uses Mobile IPv4 (Kwon et al., 2008) with MIPv6 (Kwon et al., 2008). These protocols explain the two methods which offer services of multicasting to mobile nodes. One of those is a foreign agent based multicasting referred as remote-subscription and the other is a bidirectional tunneling which is a home agent-based multicast (Xylomenos and Polyzos, 1997; Chikarmane et al., 1998). As regards to MIPv6 an ordinary change has been made to focus mainly on multicast deliverance. In other words, a moving node can decide its present location by receiving the router information messages and gather the previous path information. If the previous path information of a mobile node mismatches with the mobile host's key address then the node is said to be in a foreign network.

In IP-based wireless networks (Krishna et al., 2009a; Pack and Choi, 2003), handover latency is one of the important problems to reduce time period. MIPv6 defines how mobile host can retain its connectivity to the internet, when the node switches between the Access Routers (AR) (Yang and Chen, 2008). There is a possibility of connection switching delay as well as consideration of IP protocol operation, occurring during the handover. Due to this, handover latency occurs when there is lacking ability to send or receive packets to and from the mobile host. In many situations, the handover latency speed is higher compared to real-time applications. But this rate of latency could be adequate to maintain MIPv6 handover. Hence, an improved MIPv6 specification called Fast Handover (Koodli and Perkins,
2001; Banerjee and Dutta, 2010) is to cut down handover latency, which has been proposed by the IETF.

In wireless Internetworking, Fast handover provides faultless handover using a layer 2 trigger concept. Several studies have been done (Koodli and Perkins, 2001; Costa et al., 2002) in various networking environments to assess the performance of Fast handover. Compared to other mobile internet protocols cited in (Kwon et al., 2008), FMIPv6 offers a quicker handover process. In Hierarchical MIPv6 different hierarchies of mobility agents (Costa et al., 2002) are allowed to use this approach. An accomplishment of experiment study on handover latency showed that for each protocol the best performance could be obtained by implementing both HMIPv6 and FMIPv6.

Our main focus is to think about the difficulties of decreasing packet loss during MIPv6 fast handovers. In MIPv6, it may take a few seconds to perform handover, but it consumes more time to lose many packets, which is shown in (Kwon et al., 2008; Nguyen, 2008). In spite of multicast subscription (Kwon et al., 2008) techniques, the handover latency has an interrupted multicast packet transfer approach. The FMIPv6 key factor is to lower the MIPv6 handover latency. But there are still some unsolved problems, raised during the transmission of multicast packets. To overcome these issues, they projected efficient multicast routing protocols which do the operations of protocol, which are attained by message transmissions of FMIPv6. (Costa et al., 2002; Suh et al., 2001; Louvros, 2007) compared different mobility management schemes cited in (Yang and Chen, 2008), which are currently being conferred inside the IETF (Kwon et al., 2008).

In the previous studies, survey was done to analyze the performance of packet registrations before and after the packet delivery in handover protocols. As an outcome, the assessment of packet delay and packet loss is suggested through an easy systematic approach. Additionally with the help of above mentioned approaches, a Join operation is introduced. This Join operation consumes more time while transmitting any packet from source to destination.

To avoid Join operation completely we would like to introduce one message exchange after Handover acknowledgement which is Fast Join (F-Join). This acknowledgment makes the packet delivery with low handover latency compared to previous approach with the concept of tunneling.

Working principle of fast handover: The figure cited from (Pack and Choi, 2003) shows packet transmission in MIPv6. MIPv6 is a protocol developed as a part of IPv6 to maintain mobile connections. In the fast 
handover mechanism, Mobile-initiated Handover (MH) is linked between mobile node's Previous AR (PAR) (Yang and Chen, 2008) and New AR (NAR) (Yang and Chen, 2008). In FMIPv6 when MH is connected to the NAR, mobile node has to get hold of a new Care of Address (CoA) (Kwon et al., 2008). On receipt of new $\mathrm{CoA}, \mathrm{MH}$ has to send a Binding Update (BU) (Yang and Chen, 2008) message to mobile node's PAR. Now PAR initiates the process of sending packets to the NAR having MH's address. This handover procedure is initiated with the support of link layer (Layer 2) (Pack and Choi, 2003) information, by either MH or PAR. L2 information gives the actual location of MH. During handover procedure, Router Solicitation for Proxy (Yang and Chen, 2008) (RSP) message and Proxy Router Advertisement (Yang and Chen, 2008) (PRA) message are channeled from $\mathrm{MH}$ to PAR and PAR to $\mathrm{MH}$ respectively. After confirming from the NAR about MH information, it will send NAR Handover Initiate (Yang and Chen, 2008) (HI) message to start the bidirectional tunneling process between PAR and NAR.

In response to this, NAR sends Handover Acknowledgement (HAck) message. Before disconnecting MH's link, mobile node has to send Fast Binding Update (Kwon et al., 2008) (FBU) information to confirm whether requested handover is accepted by NAR or not. This can be checked from the status code of HAck message. Now by sending Fast Binding Acknowledgement (Koodly, 2005) (FBAck) to MH, packets are forwarded from Previous CoA (PCoA) to the NAR. Once the connection is confirmed, the MN sends a Router Solicitation Message (Koodly, 2005) (RSM) together with the Fast Neighbor Advertisement (Koodly, 2005) (FNA) message to NAR and vice versa. On successful transmission of RSM and FNA, a Router Advertisement (Koodly, 2005) (RAdv) message with the Neighbor Advertisement Acknowledgment (Koodly, 2005) (NAAck) message is also exchanged to initiate the process of distributing the buffered packets tunneled from PAR to CN.

Basically in MIP, during handover procedure there is a possibility of experiencing service interruption. This interruption extends till the HA gets the current location of MH, when MH enters into NAR. During this period, fast handover expects L3 (Pack and Choi, 2003; Banerjee and Dutta, 2010) (Network Layer) handover procedures before the L2 handover procedure get to be completed. Due to this, fast handover gives the assertion of minimum handover delay in L3 and elimination of service interruption period.

\section{MATERIALS AND METHODS}

Modification in the Existing FMIPv6: In the existing protocol (Kwon et al., 2008), focal point on mobile multicast routing is to reduce the multicast delivery. In MIPv6, minimizing the multicast packet loss is raised by collapse in the handovers. Though it is clearly not affirmed in (Nguyen, 2008), diminishing in multicast interruption period occurring during FMIPv6 handover is based on multicast subscription methods (Kwon et al., 2008). In the FMIPv6, by applying bidirectional tunneling the MN's multicast routing delay is reduced. The packets transmitted to MN's PCoA and NCoA should be encapsulated by HA and PAR of MN. As a result of this technique, the multicast routing path is lengthy. Further encapsulating each and every packet becomes a must. Besides, this approach creates many copies of multicast packets in FN, which is expensive.

As discussed in (Kwon et al., 2008), even though packet loss is minimized, still it occurs during the Join operation in FMIPv6 handover procedure. Due to the inefficiency of packet forwarding, access router is unable to transmit the packets during bidirectional tunneling approach. The suggested change required in this protocol overcomes the problem of more packet loss by doing a tiny modification in FMIPv6 protocol. The following Fig. 1 shows the modification in FMIPv6 protocol.

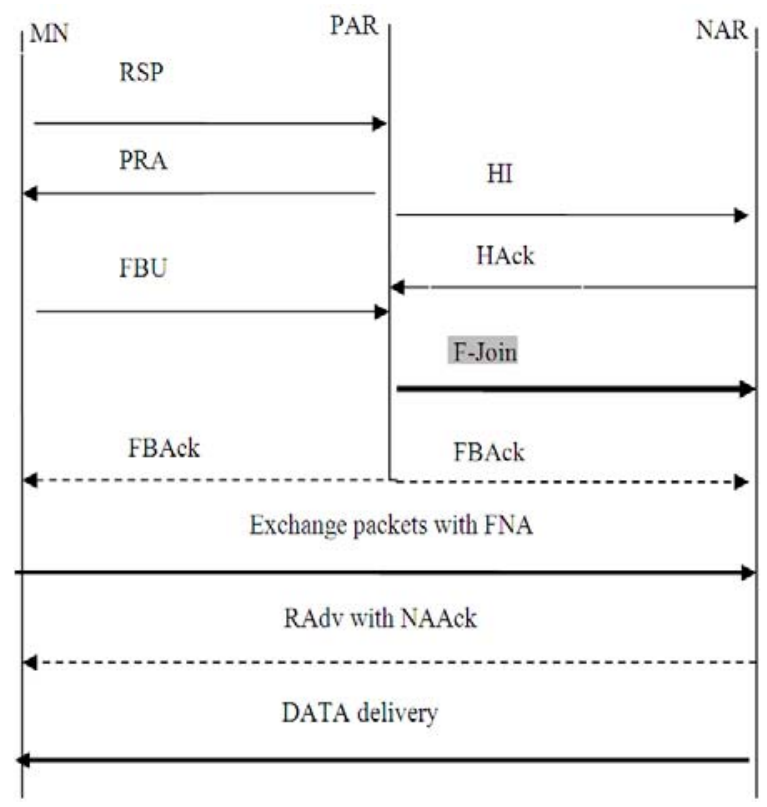

Fig. 1 F-Join operation in FMIPv6 
Before the tunneling process is gets initiated, one message exchange was introduced immediately after Hack, which is referred as Fast Join (F-Join). Because NAR takes few seconds to perform join operation to multicast each packet. And also it consumes more time to subscribe multicast group for a first time. F-join is sent from PAR to NAR in order to notify it to perform join operation for a multicast group which is already mentioned in HI. If NAR has performed a join operation already, then NAR simply ignores the F-Join. By introducing F-Join, join latency problem can be avoided and the packet loss could also be decreased. The following algorithm gives the operations of MN, NAR and PAR with F-Join in the FMIPv6 protocol.

Handover between NAR and PAR with Join operation before tunneling: // It is assumed that NAR and PAR supports FMIPv6 protocol:

Step 1: $\quad$ MN remits FBU message to PAR.

Step 2: PAR transmits F-Join message to NAR.

Step 3: If message is accepted then PAR gets FBAck with positive F-Join as an acknowledgement.

Step 4: Else FBAck with negative acknowledgement is received. // end of if

Step 5: Check whether NAR has received join operation already, if true flag is set.

Step 6: $\quad$ Otherwise Step 6 and 7 are done

Step 7: Assume NAR has not joined multicast group. Then the flag is reset.

Step 8: Now Join process is initiated. // end of if

Step 9: If FBAck receives with negative acknowledgement during F-Join operation then perform step 10 and 11

Step10: Flag is not checked

Step11: Simply perform the process of Join operation. Step12: // end of if

Step13: Now packets are transmitted from PAR to NAR along with FNA.

Step 14: On reception of packets, group id is checked.

Step 15: If id exits (flag is set), then remove the tunnel and packet is delivered.

Step 16: // end of if

Step 17: If delivered successfully then send response to PAR.

Step 18: Otherwise error message is created in NAR and send to PAR through tunnel.

Since the joining operation is carried out before L2, the remaining process of packet transmission cited in (Kwon et al., 2008) can be omitted. We tried to explain that the packet loss during fast handover can be reduced, by introducing a fast join operation before encapsulating the packets. This made us to ascertain that the handover latency problem can be minimized without incurring more loss to the packets.

\section{RESULTS AND DISCUSSION}

Performance Analysis of FMIPv6 with F-Join: This segment explicates the suggested FMIPv6 performance analysis with the elimination of Join operation in the existing scheme. The service interruption period during the handover procedure occurs due to more time consumption of a MN to inform the HA its location. Actually it occurs when MN moves between two ARs, which could be minimized using the F-Join operation before tunneling the packet. The service disruption period is an amalgamation of Link switching delay $\left(T_{L D}\right)$ between the nodes, time taken to get IP connection $\left(\mathrm{T}_{\mathrm{IPC}}\right)$ and time taken to receive a packet $\left(\mathrm{T}_{\mathrm{PR}}\right)$. Link switching initiates the process of Handover. IP connectivity is to locate a neighbor node by waiting for an acknowledgement from a binding update message. Packets are received when they start arriving at the new IP address. Based on these parameters the service interruption can be calculated as:

$\mathrm{T}_{\mathrm{SI}}=\mathrm{T}_{\mathrm{LD}}+\mathrm{T}_{\mathrm{IPC}}+\mathrm{T}_{\mathrm{PR}}$

After introducing F-Join operation, IP connectivity latency is almost reduced totally as the time required for receiving a packet consumes same quantity time for IP connection. So the service delay can be reduced by eliminating IP connectivity time. This can be attained by using the following formula:

$\mathrm{T}_{\mathrm{SI}}=\mathrm{T}_{\mathrm{LD}}+\mathrm{T}_{\mathrm{PR}}$

The equation (2) shows that the time taken to initiate the process of handover is reduced. During this initiation process MN could lose its' connectivity due to a sudden degradation of a link quality. The packet forwarding during this period has less packet loss. In other words, F-Join in FMIPv6 has very less or no packet loss arriving during this period. The overall packet delivery rate is calculated from the packet transmission cost and packet loss rate occurring during handover procedure of FMIPv6. Therefore, the proposed FMIPv6 with F-Join can lessen the anticipated handover latency.

\section{CONCLUSION}

FMIPv6 aims to improve the handoff latency by minimizing and eliminating the disruption occurring 
during handover. This is for establishing new communication paths from the mobile node new access router without packet loss. As per our research, no developmental approach had been made for any further implementation. Thus, the service establishment to a fresh point of connection through the Fast Join operation will be faster. The new temporary address is given even before the mobile node is on its new attachment point. The mechanism discussed above will make less packet loss with the continuity of connection in the specified transmission time.

However, this proposed approach achieved with fewer nodes and can be extended with more nodes to reduce total overhead cost. This cost can be minimized in consideration with delay and latency occurring during handover. In other words the latency problem could be cut down by having minimum link switching delay, IP connectivity delay and packet reception latency. A new care of address validation will be conceded by applying mobile location operation for both parent and new access points. To avoid the same IP address usage by mobile nodes, the validation can be carried out in new access router.

\section{REFERENCES}

Alfawaer, Z.M., G. Hua and N. Ahmed, 2007. A novel multicast routing protocol for mobile ad hoc networks. Am. J. Applied Sci., 4: 333-338. DOI:10.3844/ajassp.2007.333.338

Banerjee, A. and P. Dutta, 2010. Link stability and node energy conscious local route-repair scheme for mobile ad hoc networks. Am. J. Applied Sci., 7: 1139-1147. DOI:10.3844/ajassp.2010.1139.1147

Chikarmane, V., C.L. Williamson, R.B. Bunt and W.L. Mackrell, 1998. Multicast support for Mobile hosts using mobile IP: design Issues and proposed architecture. Mobile Netw. Appli., 3: 365-379. DOI: 10.1023/A:1019101521453

Costa, X.P., R. Schmitz, H. Hartenstein and M. Liebsch, 2002. A MIPv6, FMIPv6 and HMIPv6 handover latency study: Analytic approach. Proceeding of the Ist Mobile and Wireless Telecommunications Submit, (MWTS'02), NEC Europe Ltd, Germany, pp: 100-105.

Koodli, R. and C.E. Perkins, 2001. Fast handovers and context transfers in mobile networks. ACM Comput. Commun. Rev., 31: 37-47. DOI: 10.1145/1037107.1037113

Koodly, R., 2005. Fast handovers for mobile IPv6. The Internet Society. http://www.faqs.org/rfc/rfc4068.txt
Kwon, D.H., W.J. Kim and Y.J. Suh, 2008. An Efficient Mobile Multicast mechanism for fast handovers: a study from design and implementation in experimental networks. Compu. Communi., $\quad 31$ : 2162-2177. DOI: 10.1016/j.comcom.2008.02.008

Louvros, S., 2007, Handover outage ttime optimization in heterogeneous networks. J. Comp. Sci., 3: 860862. DOI: $10.3844 /$ jcssp.2007.860.862

Nguyen, U.T., 2008. On multicast routing in wireless mesh networks. Comput. Communi., 31: 13851399. DOI: 10.1016/j.comcom.2008.01.022

Pack, S. and Y. Choi, 2003. Performance analysis of fast handover in mobile ipv6 networks. Lec. Not. Comput. Sci., 2775: 679-691. DOI: 10.1007/978-3540-39867-7_64

Paul, S., 1998. Multicasting on the Internet and Its Applications. 1st Edn., Springer, USA., pp: 456. ISBN: 0792382005

Gattupalli, P. and P.V. Krishna, 2010. A framework for Qos multicast videostreaming in wireless mesh network. Int. J. Adv. Comput. Communi., 2: 13-13.

Suh, Y.J., H.S. Shin, D.H. Kwon, 2001. An efficient multicast routing protocol in wireless mobile networks. Wireless Netw., 7: 443-453. DOI: 10.1023/A:1016780525280

Krishna, V.P., S. Misra, M.S. Obaidat and V. Saritha, 2009a. An efficient approach for distributed dynamic channel allocation with queues for realtime and non-real-time traffic in cellular networks. J. Sys. Software, 82: 1112-1124. DOI:10.1016/J.JSS.2009.01.043

Xylomenos, G. and G.C. Polyzos, 1997. IP multicast for mobile hosts. IEEE Commun. Maga., 35: 5458. DOI: $10.1109 / 35.568210$

Yang, D.N. and M.S. Chen, 2008. Efficient resource allocation for wireless multicast. IEEE Trans. Mobile Comput., 7: 387-400. DOI: 10.1109/TMC.2007.70739 